\title{
Identifying Modes in a Pulsating sdB (EC 14026) Star
}

\author{
M.D. Reed
}

Southwest Missouri State University, Department of Physics, Astronomy, and Material Science, Sprintfield, MO, 65804, e-mail: mdr131f@smsu.edu

Iowa State University, Department of Physics and Astronomy, Ames, IA. 50011, USA

and the Whole Earth Telescope Xcov 17 and 21 teams $^{2}$

\begin{abstract}
We present a preliminary analysis of time series photometry on the pulsating subdwarf B star PG 1336-018 acquired as part of two Whole Earth Telescope runs. PG 1336-018 is a multi-mode pulsator with at least 15 periods centered around 175 seconds. It is also an HW Vir-type eclipsing binary with a period of 2.4 hours. Although over 20 pulsating subdwarf B stars have been discovered, only a few have resolved temporal spectra and still fewer have identified pulsation modes. We analyze features unique to the eclipsing binary system of PG 1336-018 as a means of identifying pulsation modes.
\end{abstract}

\section{Introduction}

Pulsating sdB (also known as sdBV or EC14026) stars offer us the potential to probe the interior of Blue Horizontal Branch (or sdB for field) stars using asteroseismology. PG 1336-018 (hereafter PG 1336) was discovered to be a pulsating sdB star by Kilkenny et al. (1998), with the added bonus that it is also an eclipsing binary. The companion is a late-type $\sim \mathrm{M} 5$ dwarf, so the stellar radii are comparable, yet the companion contributes little to the integrated flux. During primary eclipse the companion covers about half of the pulsator.

\section{Previous studies}

PG 1336 was the target of a Whole Earth Telescope (WET) campaign in April of 1999. It was observed to pulsate in at least 15 modes (Reed et al., 2000) with 2 modes split by twice the orbital frequency. We wished to use the eclipse to identify some of the remaining modes.

Pulsations manifest themselves as alternating hot and cool regions on the star's surface. As the visible surface area changes (during eclipse), so should the observed pulsation amplitudes. An analysis in which the pulsation axis

\footnotetext{
${ }^{2} \mathrm{~A}$ complete list of participants is available at $\mathrm{http}: / /$ wet.iitap.iastate.edu
} 
was aligned with the rotation axis was presented in Reed et al. (2001). They determined that modes with $\ell=1, m=0 ; \ell=2, m= \pm 1$; and all $\ell=3$ modes would only be visible during eclipse while $\ell=2, m= \pm 2$ modes would disappear during eclipse. Other modes would remain unaffected. This means eclipses could be used to identify pulsation modes.

Unfortunately, Xcov 17 did not have the usual WET coverage and was not optimized to study the eclipses. However it did prove the method to be viable and, in April, 2001, the WET observed PG 1336 again.

\section{3. $X \operatorname{cov} 21$}

During Xcov 21, 440 hours of data were collected from April 17-30, 2001 (a duty cycle of $\sim 62 \%$ ). Over 27 separate modes were identified in the out-of-eclipse (OoE) temporal spectrum and this time the data were optimized to examine the pulsations through eclipse. Although the task was still non-trivial, we were able to detect 15 peaks. As summarized in Sect. 2, we would expect to observe several modes in both the OoE and primary eclipse data, as well as new, rotationally split modes in the primary eclipse data. This was not the case. Of the 15 modes detected during primary eclipse, only 2 were observed in the OoE data (the 2 with the largest amplitudes).

Our first assumption was that the primary eclipse data were too contaminated with aliases to be useful. To test this, we reduced and analyzed data from the secondary eclipse in the same fashion as from the primary eclipse. We readily detected 12 peaks on the temporal spectrum, 11 of which coincided exactly (to within the errors) with those detected in the OoE data. This gave us confidence that it was not a reduction problem. It more likely indicates that aligning the pulsation axis with the rotation axis is a poor assumption.

\section{A tipped pulsation axis}

As first suggested by Margarida Cunha (private communication), if the tidal force dominates the Coriolis force, the pulsation axis may align along the axis of the binary. This is similar to the roAp stars, where the magnetic field dictates the alignment of the pulsation axis. To determine the effect of such an alignment, we created simulated light curves where the pulsation axis is tipped by 90 degrees (the inclination of the actual binary is 81 degrees) and precesses to always point toward the companion (eclipses were ignored). Our simulated light curves and Fourier transforms are provided in Fig. 1. Pulsation modes are indicated on the left of each plot (as in $\mathrm{f} \ell m$ ). For the light curves, phases (where a shift of $0.5=180^{\circ}$ ) are dashed lines, with scales on the right of the plot and dotted lines indicate orbital phases ( $\mathrm{PE}=$ primary eclipse, $\mathrm{SE}=$ secondary eclipse, $\mathrm{T}$ and $A=$ quadrature when the star is moving toward and away from the observer, respectively). The dot-dashed line indicates the input frequency (corresponding to a pulsation period of 175 seconds) of the FT, the dotted line is $1 \cdot f_{\text {orb }}$ away from the input signal and the dashed line is $2 \cdot f_{\text {orb }}$ away from the input signal. As indicated in the figure, every non-radial mode undergoes at least two $180^{\circ}$ shifts in phase during each orbital cycle. 

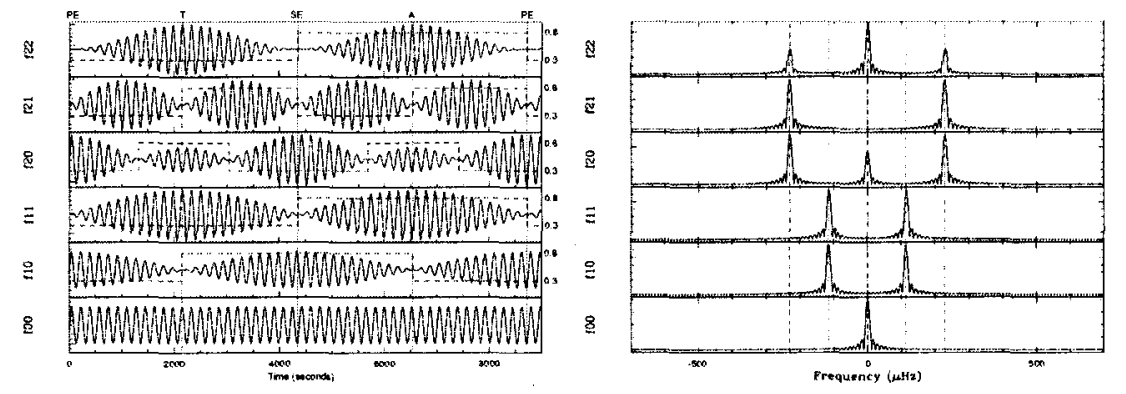

Figure 1. Left: Simulated light curves and FTs with the pulsation axis tipped 90 degrees to the rotation axis.

Each pulsation frequency produces a complex light curve which creates a many-peaked temporal spectrum. But this complexity may be the key to understanding the pulsations. PG 1336 has many peaks in the temporal spectrum; by tipping the pulsation axis, we can create many peaks from fewer actual pulsations. If the lightcurve is binned by orbital phase for a particular mode, then only the real peak will be present, and predictable phase shifts for similar valued modes will be observed.

Of the 4 highest amplitude modes, two are split by $2 \cdot f_{\text {orb }}$, a signature of $\ell=1$ modes. We divided up the light curve and binned by orbital phase appropriate for an $\ell=1, m= \pm 1$ mode. We then analized each temporal spectrum independently. In both (phase) cases, a peak midway between appeared where none previously existed in the combined spectrum. Additionally, the phase between the regional spectra changed, as expected for an $\ell=1, m= \pm 1$ mode. This provides strong indications that indeed PG 1336 has a tipped pulsation axis. The next step will be to bin the light curve by orbital phase appropriate for other modes and perform similar tests. Such tests will be performed in the near future.

\subsection{Eclipses revisited}

Simulations like those in Reed et al. (2001) were created to model light curves during primary eclipse for the re-aligned pulsation axis. With the exception of the $\ell=2, m= \pm 2$ modes, all modes show no effect from the eclipse in phase and only mild changes in amplitude. Thus, if the pulsation axis is tipped 90 degrees to the rotation axis, amplitude during the primary eclipse is no longer an indicator of pulsation modes.

\section{Conclusions and future work}

Figure 2 shows the frequencies detected from various reductions of the data for PG 1336 from the Xcov 21 WET run. More than 20 individual frequencies are detected in the combined data set, most of which are detected in other, regional data sets as well. The primary eclipse temporal spectrum is the most incongruent, with only 2 modes detected in other temporal spectra. Many of 


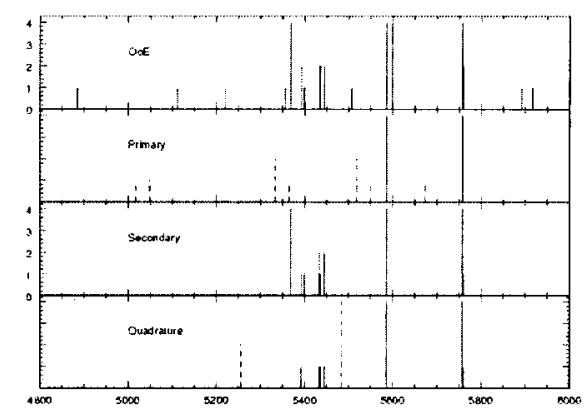

Figure 2. Frequencies detected in the temporal spectra of PG 1336 binned in amplitude (for clarity). Solid lines are modes present in the OoE temporal spectrum while dashed lines indicate peaks only observed in the labeled temporal spectra.

the remaining modes are orbital separations away from modes detected in the OoE spectrum.

The temporal spectra at eclipse and quadrature provide good indications that the pulsation axis of PG 1336 is not aligned with the rotation axis, but rather (at least somewhat) with the tidal force from the companion. This likely has far broader applications than just PG 1336. Since a sizable fraction of sdB stars are likely in binaries (Green et al., 2001), it is probable (and almost a certainty for KPD $1930+2752$ ) that other pulsators also have pulsation axes which (somewhat) align with the tidal force of their companions.

I would also like to call on theorists to help in understanding how pulsations respond to tidal forces. It is already believed that $p$ and $g$ modes will "feel" the tidal forces differently, but it would be helpful for observers to have some form of parameterization so we can predict what the pulsation axis should be. There is also some question (at least in my mind) as to what it means to have $m \neq 0$ modes which are not due to rotation.

\section{References}

Green, E.M., Liebert, J., \& Saffer, R.A. 2001, in ASP Conf. Ser., Vol. 226, On The Origin of Subdwarf B Stars and Related Metal-Rich Binaries, eds. J. Provencal et al., (San Francisco ASP), 192

Kawaler, S.D 1999, in ASP Conf. Ser., Vol. 169, 11th European Workshop on White Dwarfs, eds. S.-E. Solheim \& E. Meistas, (San Francisco ASP), 158

Kilkenny, D., et al. 1998, MNRAS, 296, 329

Reed, M.D., et al. 2000, BaltA, 9, 211

Reed, M.D., Kawaler, S.D., \& Kleinman, S.J. 2001, in ASP Conf. Ser., Vol. 226, On The Origin of Subdwarf B Stars and Related Metal-Rich Binaries, eds. J. Provencal et al., (San Francisco ASP), 181 